\title{
Alpha-particle radiotherapy: For large solid tumors diffusion trumps targeting
}

Charles Zhu ${ }^{1}$, Michelle Sempkowski ${ }^{1}$, Timothy Holleran ${ }^{1 \dagger}$, Thomas Linz ${ }^{2 \dagger}$, Thomas

Bertalan $^{3}$, Anders Josefsson ${ }^{4}$, Frank Bruchertseifer ${ }^{5}$, Alfred Morgenstern ${ }^{5}$, Stavroula Sofou $^{1,2,6^{*}}$

running title: Targeted versus non-targeted $\alpha$-particle therapy for solid tumors 
Diffusion limitations on the penetration of nanocarriers in solid tumors hamper their therapeutic use when labeled with $\alpha$-particle emitters. This is mostly due to the $\alpha$ particles' relatively short range $(\leq 100 \mu \mathrm{m})$ resulting in partial tumor irradiation and limited killing.

To utilize the high therapeutic potential of $\alpha$-particles against solid tumors, we designed non-targeted, non-internalizing nanometer-sized tunable carriers (pH-tunable liposomes) that are triggered to release, within the slightly acidic tumor interstitium, highly-diffusive forms of the encapsulated $\alpha$-particle generator Actinium-225 $\left({ }^{225} \mathrm{Ac}\right)$ resulting in more homogeneous distributions of the $\alpha$-particle emitters, improving uniformity in tumor irradiation and increasing killing efficacies.

On large multicellular spheroids (400 $\mu$ m-in-diameter), used as surrogates of the avascular areas of solid tumors, interstitially-releasing liposomes resulted in best growth control independent of HER2 expression followed in performance by (a) the HER2targeting radiolabeled antibody or (b) the non-responsive liposomes. In an orthotopic human HER2-negative mouse model, interstitially-releasing ${ }^{225}$ Ac-loaded liposomes resulted in the longest overall and median survival.

This study demonstrates the therapeutic potential of a general strategy to bypass the diffusion-limited transport of radionuclide carriers in solid tumors enabling interstitial release from non-internalizing nanocarriers of highly-diffusing and deeper tumorpenetrating molecular forms of $\alpha$-particle emitters, independent of cell-targeting. 
keywords: interstitial transport; pH-responsive liposomes; $\alpha$-particle therapy; Actinium225; tumor penetration; solid tumors 


\section{Introduction}

In solid tumors the diffusion-limited penetration depths of radionuclide carriers [1-3] combined with the short range of $\alpha$-particles $(40-100 \mu \mathrm{m})$ [4] hamper their use mostly due to partial tumor irradiation. Given the high killing efficacy of $\alpha$-particles (4-5 tracks across the nucleus result in cell kill) [5-11] which is also mostly independent of celloxygenation state unlike $\beta$-particles [12] this is quite unfortunate. Carrier molecules that diffuse substantially from the vasculature into the viable tumor have been suggested to be necessary for effective irradiation of the viable solid tumor-cell regions [3].

To address the diffusion-limitations on the transport of radionuclide carriers and the partial tumor irradiation, we designed nanometer-sized lipid carriers (liposomes) that are triggered to release in the tumor interstitium highly-diffusing forms of encapsulated $\alpha$ particle emitters. Given that the molecular-size of the released chelated-emitters is significantly smaller than the nanometer-sized carriers, we expect that the corresponding higher diffusivities may result in more uniform distributions and deeper penetration of radionuclides within tumors, resulting in more even tumor irradiation. Towards this goal, we designed $\mathrm{pH}$-responsive liposomes encapsulating chelated forms of $\alpha$-particle emitters. These liposomes are expected to demonstrate enhanced uptake by solid tumors as described by the Enhanced Permeability and Retention (EPR) effect [13]. Additionally, in the slightly acidic tumor interstitium $(7.4>\mathrm{pH}>6.0)[14,15]$ a $\mathrm{pH}-$ responsive mechanism on the liposome membrane results in release of these chelated forms of encapsulated radioactivity [16]. 
The $\mathrm{pH}$-triggered release mechanism is simply based on the formation of defective interfaces - in terms of lipid packing - that span the lipid bilayer resulting in high membrane permeability $[16,17]$. These lipid interfaces originate at the boundaries of domains formed upon lipid phase separation. Liposome membranes are designed to contain two lipid types: one with a non-titratable headgroup (lipid with gray headgroup in cartoon, Figure 1) and one with a titratable domain-forming headgroup (lipid with red headgroup); the tail lengths are different to enhance lipid packing defects at the interfaces upon phase separation [16,17]. The extent of ionization on the headgroups of the domain-forming lipids is controlled by the $\mathrm{pH}$ which is used to shift the balance between electrostatic repulsions and H-bonding attractions [18]. At neutral $\mathrm{pH}$, the headgroups of the domain forming lipids are negatively-charged, opposing close proximity between lipids, resulting in uniform lipid membranes. As the $\mathrm{pH}$ is decreased, gradual headgroup protonation minimizes the electrostatic repulsion, and phase separated lipid domains are formed driven mostly by H-bonding [19]. The utility and robustness of this approach has been reported for several agents in vitro and in vivo $[16,20-22]$.

In this study, pH-responsive (interstitially-releasing) liposomes were loaded with the $\alpha$ particle generator Actinium-225 $\left({ }^{225} \mathrm{Ac}\right)$ and were compared to non-pH-responsive (non-releasing) liposomes and to radiolabeled antibodies (Trastuzumab) targeting HER2. We evaluated the distributions of the carriers, and of the delivered radioactivity, in multicellular spheroids comprised of breast cancer cells with variable radiosensitivities and variable expression levels of HER2. Lastly, the efficacy of all 
constructs to prolong survival was evaluated on animals with orthotopic MDA-MB-231 triple negative breast cancer (TNBC) xenograft tumors. 


\title{
Materials and Methods
}

\section{Materials}

The lipids 1,2-dihenarachidoyl-sn-glycero-3-phosphocholine (21PC), 1,2-distearoyl-snglycero-3-phosphate (DSPA), 1,2-distearoyl-sn-glycero-3-phosphoethanolamine-N[methoxy(polyethylene glycol)-2000] (DSPE-PEG), 1,2-dipalmitoyl-sn-glycero-3phosphoethanolamine-N-(lissamine rhodamine B sulfonyl) (Rhd-lipid) were purchased in chloroform from Avanti Polar Lipids (Alabaster, AL). Poly(2-hydroxyethyl methacrylate) (polyHEMA) was obtained from Polysciences, Inc. (Warrington, PA). All materials are described in detail in the supplemental data. The IgG1K isotype control antibody was purchased from SouthernBiotech (Birmingham, AL). Actinium-225 $\left({ }^{225}\right.$ Ac, actinium chloride) was provided by the Institute for Transuranium Elements, Germany, as described before [23,24].

\begin{abstract}
Nanocarriers
$\mathrm{pH}-$-responsive and non-pH-responsive liposomes were composed of 21PC:DSPA:cholesterol(chol):DSPE-PEG:Rhd-lipid at a mole ratio of 53.7:23.0:9.5:13.0:0.8 and of 21PC:chol:DSPE-PEG:Rhd-lipid at a mole ratio of 66.0:28.0:5.0:1.0, respectively, and were prepared using the thin film hydration method [25]. Lipid suspensions were extruded through $100 \mathrm{~nm}$ diameter pores, and were characterized for size and zeta potential using a Zetasizer NanoSeries (Malvern Instruments Ltd.). Liposomes encapsulating 1,4,7,10-tetraazacyclododecane-1,4,7,10tetraacetic acid (DOTA) were loaded using the A23187 ionophore [26]. Loading efficiency was determined by comparing the Bismuth-213 $\left({ }^{213} \mathrm{Bi}\right) \gamma$-emissions $(360-480$
\end{abstract}


$\mathrm{keV}$ ) at secular equilibrium before and after Sephadex-G-50 separation using a Cobra $\gamma$ counter (Packard Instrument Co., Inc.).

To radiolabel antibodies with ${ }^{225} \mathrm{Ac}[23,24]$ or Indium-111 $\left({ }^{111} \mathrm{In}\right)$, the isothiocyanatefunctionalized metal chelator (DOTA or diethylenetriamine pentaacetic acid (DTPA), respectively) was added in one step as described in supplemental data [27]. Radiochemical purity was assessed via instant thin layer chromatography (iTLC). The

${ }^{111}$ In-DTPA-Trastuzumab was used to measure the levels of HER2 receptors per cell as reported before [28,29].

Retention of ${ }^{225} \mathrm{Ac}$ was determined by incubating the ${ }^{225}$ Ac-loaded carriers in HybriCare media supplemented with $10 \%$ fetal bovine serum (FBS) at $37^{\circ} \mathrm{C}$. At pre-determined time points, aliquots were removed, and released radioactivity was separated either with a Sephadex-G-50 (liposomes) or 10-DG (antibodies) column [26].

The extents of released radioactivity from liposomes corresponding to each of the three possible forms $\left({ }^{225} \mathrm{Ac}-\mathrm{DOTA},{ }^{225} \mathrm{Ac}\right.$-ionophore complex, $\left.{ }^{225} \mathrm{Ac}\right)$ were evaluated by separation and quantitation of two of the forms in addition to the overall radioactivity balance (as described in detail in supplemental data).

\section{Cell and Spheroid Culture}

The HER2 positive BT474 cell line, the TNBC MDA-MB-231 cell line, and the HER2negative MCF-7 cell line which was stably transfected (by Dr. V. Karantza) to express moderate HER2 levels were obtained from and maintained in ATCC recommended 
media supplemented with $10 \%$ FBS, 100 units $/ \mathrm{mL}$ penicillin, and $100 \mu \mathrm{g} / \mathrm{mL}$ streptomycin at $37^{\circ} \mathrm{C}, 5 \% \mathrm{CO}_{2}$. MCF-7/HER2 media was also supplemented with 2 mg/mL G418 antibiotic. The expression of HER2 per cell was evaluated using ${ }^{111} \mathrm{In}$ DTPA-Trastuzumab by measuring the extent of cell-associated antibodies for fixed cell density while varying the antibody-to-cell ratios at $4^{\circ} \mathrm{C}$, and by correcting for nonspecific binding at 50x excess of non-radiolabeled antibody and for its immunoreactivity, repeated at two different cell densities [29].

Spheroids were initiated by seeding 400 (BT-474) or 250 (MCF-7/HER2) cells/well into polyHEMA-coated 96-well plates with U-shaped wells or by mixing 125 (MDA-MB231) cells/well with $2.5 \% \mathrm{w} / \mathrm{v}$ Matrigel ${ }^{\mathrm{TM}}$-containing media followed by centrifugation for 5 minutes at 500x gravity, and incubation until spheroids grew to appropriate size (typically at least one week). The number of cells per spheroid were counted following dissociation (by incubation with trypsin) of the corresponding spheroids.

\section{Measurement of Spheroid Interstitial pH-Gradients}

The $\mathrm{pH}$ gradients of the interstitium of spheroids were determined by incubating overnight - spheroids with the pH-responsive membrane-impermeant fluorophore SNARF-4F [22,30], and, upon transfer to fresh media, by acquiring optical sections through the spheroid equator using a Leica TCS SP2 confocal laser scanning microscope under a 10x, 0.4 NA dry objective. The ratio of emitted fluorescence intensities at $640 \mathrm{~nm}$ over the intensities at $580 \mathrm{~nm}(\mathrm{ex}: 514 \mathrm{~nm})$ - which is a function of $\mathrm{pH}$ and is independent of the concentration of the fluorophore [30] - was used to quantitate the $\mathrm{pH}$ in the spheroid interstitium using a calibration curve of the fluorophore in media at known $\mathrm{pH}$ 
values acquired at identical imaging conditions in the absence of spheroids. To evaluate the radial $\mathrm{pH}$ gradient of the spheroid interstitium, an eroding algorithm was applied on spheroid images at progressively smaller radii and, within each ring ( $6 \mu \mathrm{m}$ thickness), the average measured pixel intensity was calculated and compared to the calibration curve.

\section{Nanocarrier Profiles in Spheroids}

Following incubation with FITC-labeled antibodies (see supporting information for antibody labeling) or Rhd-lipid-labeled liposomes, spheroids were either washed and incubated in fresh media (clearance studies), or were immediately placed into optimal cutting temperature (OCT) compound following freezing and cryosectioning into $20 \mu \mathrm{m}$ thick slices. Slices were then imaged using transmission fluorescence microscopy by an Olympus IX70 inverted microscope (Olympus America Inc, PA) with a 20x, 0.25 NA dry objective. The radial nanocarrier averaged concentrations were determined using an erosion algorithm averaging within concentric rings of 3 pixels $(2.7 \mu \mathrm{m})$ width. Calibration curves were generated by measuring the intensities of serial dilutions of known concentrations in a $20 \mu \mathrm{m}$-pathlength cuvette.

\section{Actinium-225 Profiles in Spheroids}

Spheroids were incubated with ${ }^{225}$ Ac-labeled carriers for 6 hours, briefly washed (5-10 seconds) by being transferred in fresh media and were then frozen within OCT. Eight$\mu \mathrm{m}$-thick spheroid slices were placed on a Zinc-Sulfur-Silver-containing scintillation sheet and were imaged using a digital autoradiography imaging technique ( $\alpha$-Camera) [31]. The $\alpha$-Camera calibration coefficient y $\left(\mathrm{Bq} /\left(\text { pixel intensity unit* } \mathrm{h}^{-1}\right)^{*} 10^{-9}\right)$ for equilibrium ${ }^{225}$ Ac determined using the method of Chouin et al.[32] is described in detail 
in supplemental data. In spheroids, the average activities of ${ }^{225} \mathrm{Ac}$ as a function of radial position were determined using the eroding algorithm described above (of 11-16 $\mu \mathrm{m}$ thickness rings).

\section{Phosphorylation levels of histone $\gamma-H 2 A X$ in spheroids}

One hour after completion of the 6 hour incubation with radiolabeled carriers and subsequent wash, spheroids were fixed, mounted in OCT gel, frozen, and sectioned at 20 $\mu \mathrm{m}$ thickness slices. Following permeabilization, slices were immunostained with an anti-phospho-H2AX primary antibody (Clone JBW301, $2 \mu \mathrm{g} / \mathrm{mL}$ ) followed by an AlexaFluor-555-goat anti-mouse IgG1 according to manufacturer's instructions (see details in supplemental data). Spheroid slices were imaged using fluorescence microscopy (Olympus IX81 with a 20x, 0.25 NA dry objective), and an eroding code (as above) was used to quantitate the radial average distributions of the extent of $\gamma \mathrm{H} 2 \mathrm{AX}$ phosphorylation.

\section{Cell Monolayer and Spheroid treatment with ${ }^{225}$ Ac-labeled carriers}

Cells in monolayers or in spheroids were incubated for six hours with cell culture media containing liposomes $(0.1 \mu \mathrm{mol}$ lipids) or $10 \mu \mathrm{g} / \mathrm{ml}$ Trastuzumab (100x in excess of total HER2 receptors) at different radioactivity levels, and were then washed with fresh nonradioactive media. Cell monolayers were evaluated for viability by a colony formation assay [33] and by the 3-(4,5-Dimethylthiazol-2-Y1)-2,5-Diphenyltetrazolium Bromide (MTT) assay [26] after two doubling times (one doubling time was 48, 36, and 30 hours for BT-474, MDA-MB-231 and MCF-7/HER2, respectively). Evaluation of the radiosensitivity of cells was conducted in suspension following cesium-137 irradiation 
and alpha-particle irradiation via a radiolabeled isotype control antibody as described in supporting information.

Spheroid volume was monitored until the untreated population stopped growing, or until spheroid disaggregation/cell death. Volume was determined as $V=(1 / 6) \pi\left(a b^{2}\right)$, where a and $b$ are the major and minor axes, respectively.

\section{In Vivo Evaluation}

The maximum tolerated dose (MTD) of all radioactive constructs was determined by retro-orbitally injecting healthy athymic nude female mice weighing approximately 20 grams with varying doses of radioactivity $(3.7,9.25,14.8,18.5$, and $33.3 \mathrm{kBq}$ per mouse or $100,250,400,500,900 \mathrm{nCi}$ per mouse). MTD was defined as the maximum dose at which no mouse died or required euthanasia per Institutional Animal Care and Use Committee protocol (IACUC). For efficacy studies, a small incision was made on each mouse, allowing injection of 2 million MDA-MB-231 cells suspended in serum-free cell culture media into the second mammary fat pad on the right side of animals. Radiotherapy or saline was administered retro-orbitally once tumors reached $100 \mathrm{~mm}^{3}$. Animal weight, tumor volume, and overall survival were monitored. Per IACUC protocol, animals were euthanized if they met conditions for euthanasia or if tumors exceeded $10 \%$ of the overall weight of the mouse.

Statistical analysis 


\begin{abstract}
Results are reported as the arithmetic mean of $n$ independent measurements \pm the standard deviation. Student's t-test was used to evaluate differences in behavior between constructs. Survival statistics were analyzed using log-rank testing (MedCalc).
\end{abstract}




\section{Results \\ Carriers}

Tables 1 and 2 show that radiolabeling for all carriers was sufficient and stable. In particular, liposomes were loaded with more than $43.5 \pm 12.8 \%$ of introduced radioactivity which was retained at high levels $(>77.6 \pm 6.2 \%)$ at physiologic conditions $(\mathrm{pH}=7.4,24 \mathrm{hr})$ in the presence of serum proteins. As expected, only $\mathrm{pH}-$ responsive liposomes exhibited significant release of radioactive contents at $\mathrm{pH} 6.0$ (see also Figure S1) corresponding to the mildly acidic $\mathrm{pH}$ of the tumor interstium $[14,15]$. The duration of six hours of incubation in the acidic environment was chosen to represent a conservative estimate of the liposomes' residence time in tumors in vivo. The majority $(\sim 76 \%)$ of released radioactivity from liposomes was measured to be in the form of

${ }^{225}$ Ac-DOTA chelates followed by approximately $23 \%$ in the form of ${ }^{225}$ Ac-ionophore complexes (Table 1S). The radiolabeling efficiency of antibodies was also above at least $50 \%$ of introduced radioactivity (Table 2 ) which was retained at high levels $(>82.8 \pm 5.9$ $\%)$ at physiologic conditions $(\mathrm{pH}=7.4,24 \mathrm{hr}$ ) in the presence of serum proteins. The immunoreactivity of the radiolabeled HER2-targeting antibody was also preserved at high levels $(86.5 \pm 6.2 \%$, Table 2$)$.

\section{Cell Lines and Spheroids}

The HER2 expression by cells harvested from monolayers (Table 3) followed the order: BT474 >> MCF-7/HER2 > MDA-MB-231 (also on Figures S2, S3 and S4). Only on 'large' (400 $\mu$ m-in-diameter) BT474 spheroids, the expression levels of HER2 by cells was lower than the corresponding cells in monolayers (Figure S5). The interstitial $\mathrm{pH}$ gradients of spheroids were found to depend mostly on spheroid size, and $\mathrm{pH}$ values at 
the spheroid centers were $\sim 6.4-6.5$ and $\sim 6.1-6.2$ for 200 and $400 \mu$ m-diameter spheroids, respectively (Figure S6). For BT474, $1266 \pm 89$ cells were measured per 200 $\mu \mathrm{m}$-spheroid and $9833 \pm 520$ cells were measured per $400 \mu \mathrm{m}$-spheroid, and for MDAMB-231, $1093 \pm 87$ cells were measured per $200 \mu \mathrm{m}$-spheroid and $8250 \pm 661$ cells were measured per $400 \mu \mathrm{m}$-spheroid.

Profiles of Carriers, of ${ }^{225} \mathrm{Ac}$ and of induced phosphorylation of histone $\gamma-H 2 A X$ in Spheroids

Both liposome types exhibited similar distribution profiles in same-size spheroids independent of HER2 expression levels (Figures 2A and 2B, S7 and S8) and penetrated deeper than the HER2-targeting antibody even in HER2-positive BT474 spheroids. Liposomes did not significantly associate with cells (< 0.6\%) (Figure S9). Only liposomes with the interstitially-releasing ( $\mathrm{pH}$-releasing) property of encapsulated ${ }^{225} \mathrm{Ac}$ contents resulted in almost uniform delivery of ${ }^{225}$ Ac-radioactivity levels (Figure 3, Figure S10) independent of spheroid type. These measurements were conducted on 400 $\mu$ m-in-diameter spheroids among which only the MDA-MB-231's developed a measurable necrotic core (Figure S11). As expected [34], the spatiotemporal profiles of the HER2-targeting Trastuzumab and of the associated radioactivity depended strongly on HER2-expression levels of cells forming the spheroids (Figures 2A, 2B and, S7 and S9).

In agreement with the distributions of delivered ${ }^{225}$ Ac-radioactivity, were also the fluorescence distributions attributed to $\gamma-\mathrm{H} 2 \mathrm{AX}$ foci on equatorial spheroid slices shown in Figure 4 (additional images in Figure S12). The intensities from $\gamma$-H2AX foci - which 
were treated as biomarkers of dsDNA breaks induced by $\alpha$-particles - were found to be greater close to the spheroid center when radioactivity was delivered by the $\mathrm{pH}$-releasing liposomes, independent of HER2 expression.

\section{Radiosensitivities of Cells and Killing Efficacies in the Absence of Transport Barriers}

Colony survival of cells treated with all ${ }^{225}$ Ac-labeled carriers in the absence of transportrelated barriers (Figure S13) demonstrated equal killing, independent of extracellular $\mathrm{pH}$ (7.4 vs. 6.5), except, of course, for the cases of specific targeting. The HER2-targeting radiolabeled antibody exhibited greater killing efficacy only on HER2-positive cancer cell lines, in a manner proportional to the cells' corresponding HER2 expression (Figure S13, Table S2). The relative radiosensitivity of cells in suspensions to $\alpha$-particle (and Cesium-137) irradiation was as follows: MCF-7/HER2 > MDA-MB-231 $\geq$ BT-474 (Figure S14).

\section{Spheroid Growth Control}

In the presence of transport barriers, i.e. in the presence of diffusion-limited transport, only on 'small' (200 $\mu$ m-diameter) HER2-overexpressing BT474 spheroids the targeting radiolabeled antibody resulted in better growth control (Figure 5A). On 'large' (400 $\mu \mathrm{m}$ diameter) spheroids of BT474 cells the $\mathrm{pH}$-releasing (interstitium-releasing) liposomes outperformed the targeted radiolabeled antibody (Figure 5C and Figures S15, S16 for different dose levels). On TNBC MDA-MB-231 spheroids, pH-releasing liposomes exhibited superior growth control independent of spheroid size (Figures 5B and 5D, and Figures S17, S18 for different dose levels) as confirmed by the outgrowth assay (Figure S19). At first sight, this appears to not be corroborated by the spheroid volume change 
measurements yet, this is easy to rationalize: due to the presence of Matrigel ${ }^{\mathrm{TM}}$ scaffolds - which were necessary to form MDA-MB-231 spheroids - the reported 'decreasing' spheroid volumes for dying cells were not representative. Indeed spheroids treated with pH-releasing liposomes showed the scaffolds becoming empty of cells, and the outgrowth assay on the residual Matrigel ${ }^{\mathrm{TM}}$ scaffolds confirmed lack of migrating cells (Figure S19). It is the scaffolding volume that did not shrink and not the spheroid volume. On small MCF-7/HER2 spheroids with moderate levels of HER2, the efficacies of the radiolabeled Trastuzumab and the $\mathrm{pH}$-releasing liposomes were comparable (Figures S20); however, on large spheroids, the $\mathrm{pH}$-releasing liposomes again controlled growth to a greater extent (Figure S21). Non-radiolabeled liposomes did not affect spheroid growth, and nonradiolabeled Trastuzumab affected only the BT474-spheroid growth (Figures S15, S16).

\section{In vivo Efficacy}

Although the MTD for all carriers on tumor-free mice was found to be $14.8 \mathrm{kBq}$ per 20 gr-animal (400 nCi per 20-gr-animal), the efficacy studies on mice bearing TNBC orthotopic tumors were performed at $9.25 \mathrm{kBq}(250 \mathrm{nCi})$ per 20-gr-animal because the MTD levels were found to be toxic. In particular, Figure S23_A shows H\&E stained sections of the organs of tumor-free mice injected with radioactivity at the MTD (14.8 $\mathrm{kBq}(400 \mathrm{nCi})$ per $20 \mathrm{gr}$ animal) at day 82 after administration of radioactivity delivered by liposomes and by the antibody. Histopathology findings included pneumonitis but no noteworthy renal or liver toxicity. Pulmonary inflammation may have been associated with intravascular hemolysis. In addition, there was diminished splenic white pulp. Figure S23_B shows H\&E stained sections of the organs of tumor-bearing mice injected with radioactivity at the MTD (14.8 kBq (400 nCi) per 20 gr animal) which died on days 
0 and 1 after administration of radioactivity. The histopathology revealed no renal or hepatic toxicities, but appreciable lung inflammation. The latter is possibly associated with increased RBC death. In addition, there was diminished splenic white pulp.

Figure 6 shows that following one administration the pH-releasing liposomes (at 9.25 $\mathrm{kBq}(250 \mathrm{nCi})$ per 20 -gr-animal $)$ resulted in best $(* * p$-value $<0.05)$ tumor growth control and longest median and overall survival (36 and 38 days, respectively) relative to both the non-pH-releasing liposomes ( 24 and 30 days, respectively) and the radiolabeled HER2-targeting antibody (24 and 26 days, respectively), which were indistinguishable from the group that received no treatment. During the efficacy study (at activity levels lower than the MTD), all animals were euthanized because of tumor size per IACUC protocol. Figure S23_C shows H\&E stained sections of the organs of tumor-bearing mice injected with radioactivity at $9.5 \mathrm{kBq}(250 \mathrm{nCi})$ per $20 \mathrm{gr}$ animal. Mice were euthanized on days 20 or 30 (Figure 6). No appreciable renal or hepatic toxicity was observed. Indirect signs of intravascular hemolysis was noted but not to the degree observed in mice at higher doses which resulted in death. 


\section{Discussion}

Alpha-particle emitters are not traditionally considered a viable avenue for the treatment of solid tumors mostly because of the diffusion-limited transport of radionuclide carriers in the tumors resulting in only partial tumor irradiation [3]. In this study we evaluated the therapeutic potential of $\alpha$-particle emitters on solid tumors using an engineered approach that alleviates the key factor of diffusion-limited transport. For the delivery of $\alpha$-particle emitters we designed tunable non-targeting, non-internalizing liposomes which were triggered within the acidic tumor interstitium to release highly-diffusive forms (due to their smaller size) of their radioactive contents. This approach resulted in more uniform distributions of the delivered radioactivity in solid tumor surrogates (spheroids), and in significantly enhanced radioactivity uptake towards the spheroid center, causing more uniform irradiation of the tumor analogues and better control of spheroid growth. In vivo, on an orthotopic xenograft model with low expression of a cell surface molecular marker (HER2), this approach resulted in the longest overall and median survival compared to both liposomal delivery lacking the interstitial release property and to delivery by a HER2-targeting radiolabeled antibody.

Reported therapeutic approaches using charged heavy particles include external beam

(for example ${ }^{12} \mathrm{C}$ ions [35,36]) and brachytherapy implants (for example ${ }^{224}$ Ra-loaded wires emitting $\alpha$-particles $[37,38])$. The current study describes a therapeutic approach that is administered systemically, it is expected to reach solid tumors following the EPR effect, and is designed to interstitially release highly diffusing forms of the $\alpha$-particle emitters as a response to the intrinsic - if it is the case $[15,39,40]$ - mild acidity of tumors. 
Our findings on fast and complete liposome clearance from spheroids (Figures S7, S8, S22), combined with the insignificant liposome association with any of the cell types, support our suggestion that the liposomes used in this study are non-targeting and noninternalizing. A similar assumption is proposed for the major form $(\sim 74 \%)$ of released radioactivity - the ${ }^{225}$ Ac-DOTA complexes - which did not significantly associate with cells (only $0.9 \pm 0.2 \%$ of introduced ${ }^{225}$ Ac-DOTA was found to associate with cells) and did not appear to penetrate cells at all. However, the remaining $\sim 24 \%$ of released radioactivity that was complexed with the A23187 ionophore - which permeates fluidphase lipid membranes - may have in addition exhibited diffusion across the cells' plasma membranes $\left(12.2 \pm 3.8 \%\right.$ of introduced ${ }^{225}$ Ac-ionophore was found to associate with cells). The latter chemical form of released radioactivity essentially introduces a second type of diffusing radioactive species.

On HER2-overexpressing BT474 spheroids, the interplay between diffusion and cell binding reactivity relative with the actual size for the avascular tumor analogue and with the trajectory length of emitted $\alpha$-particles was clearly demonstrated. Although for 'small' avascular tumor analogues the radiolabeled antibodies were more effective than the interstitial release approach from liposomes, for 'large' avascular tumor analogues the approach, which relies on improved interstitial diffusion of released radioactivity, was more effective than the targeted radiolabeled antibody (when compared on the same basis of exposure to radioactivity levels and time). It is not clear if this effect would be observed in vivo, given the different blood clearance kinetics between liposomes and antibodies; differences in clearance kinetics are expected to affect the corresponding penetration depths and retention times of radionuclide carriers (and/or their radionuclide 
contents) within tumors. However, three- and four-fold longer exposure of large HER2positive spheroids to the HER2-targeting antibody exhibited non uniform spatial profiles similar to those described by the binding-site-barrier-effect slightly shifted towards the spheroid center (Figure S24) [34].

The biodistributions of liposomes in mice have been published before [21] and are similar to PEGylated liposomes of the same size and overall zeta potential [41]. Liposomes accumulate mostly in the liver and spleen, and have significantly faster blood clearance times than the antibodies (with elimination half-lives almost 5 times faster than the elimination half-lives of antibodies) [42]. Radioactive contents of liposomes are expected to follow the clearance and biodistributions patterns of their carriers which would include mostly hepatic and splenic uptake for the liposomal contents [21]. The three forms of encapsulated ${ }^{225} \mathrm{Ac}$ (free ${ }^{225} \mathrm{Ac},{ }^{225} \mathrm{Ac}$-DOTA and ${ }^{225} \mathrm{Ac}$-ionophore), are not expected to be released from liposomes during their circulation in the blood. The liposomal contents are triggered to be released in the tumor interstitial space. Free ${ }^{225} \mathrm{Ac}$ has been reported to accumulate mostly in the liver, spleen and bone $[43,44]$, and the ${ }^{225}$ Ac-DOTA is expected to exhibit fast whole body clearance [43].

In this study, at the highest administered radiation doses delivered either by the liposomes or by the antibodies, common toxicity-related findings were present in the lung and spleen. Lungs had acute to chronic signs of pulmonary inflammation (pulmonary edema, type 2 pneumocyte hyperplasia, increased alveolar macrophages) likely impeded gas exchange. The spleen had increased red pulp hemosiderin accumulation suggestive of intravascular hemolysis as well as white pulp lymphoid depletion. No studied cases, even 
those at higher radioactivities, had appreciable renal toxicity. In the efficacy study presented in this manuscript, the administered dose (which was below the MTD) did not result in appreciable levels of above described findings.

During blood circulation, the systemically released daughters from the ${ }^{225} \mathrm{Ac}$ decay are not retained by the DOTA chelator neither by these 'small' liposomes [45] nor by the radiolabeled antibodies [46]. Systemic release from carriers of ${ }^{213} \mathrm{Bi}$, the last of the emitted radioactive daughters, may cause renal toxicities in mice which can be addressed with pharmacologic interventions (e.g. diuretics) [47]. We did not use diuretics in this study. In humans, the bone marrow could be the dose limiting organ upon administration of ${ }^{225} \mathrm{Ac}$ using radiolabeled antibodies [48]. Such liposome studies in humans do not yet exist.

Another potential source of toxicity may arise from the release, back into the blood stream, of a fraction of the highly-diffusing forms of radionuclides from the tumor interstitium. Although these ${ }^{225} \mathrm{Ac}$-forms are mostly chelated to DOTA, their ${ }^{213} \mathrm{Bi}$ daughters could be toxic to the kidneys, yet they should only correspond to a small fraction of the approximately $2-3 \%$ of the injected dose that usually reaches the tumors [49].

The clearance of liposomes from tumors may in principle be delayed by functionalizing the carriers with cancer-cell binding/targeting ligands [50,51]. For the approach presented herein, this functionalization should preferably not result in fast internalization of the nanocarriers by the cancer cells but should enable long binding times of the nanocarriers 
on the surface of cells. In this way, longer residence times of the nanocarriers in the tumor would be enabled possibly resulting in release of greater extents of therapeutic contents within the tumor interstitium improving efficacy.

The tumor uptake of liposomes is driven by the EPR effect which does not characterize all of human tumors [52], but should be expected to describe some of the fast growing tumors in humans [53]. Towards this goal, probe nanoparticles have been suggested to be used as biomarkers of EPR for patient selection and predictive nanomedicine [54] with some approaches already in clinical trials [55].

In vivo the applicability of this strategy is determined by the extent of acidification of the tumor interstitium which activates the release process. Such levels of acidity as the ones studied herein, and even more pronounced $(\mathrm{pH}<6.5)[15]$, have been documented for patients with different types of tumors including patients with TNBC [39,56,57]. Our approach of delivering highly-diffusing forms of $\alpha$-particle emitters within the tumors, obviating the need for targeting, exhibited better efficacy in animals bearing TNBC xenografts. We suggest, therefore, that our 'diffusion-assisted' approach may ultimately offer a therapeutic option to patients with TNBC or with other 'untargetable' cancers for which targeted therapies are simply not applicable. Finally, the impact of this approach may not only be limited to solid tumors which express low levels of known receptors, but may extend to tumors with high variability in expression of known cell surface receptors, for which targeted approaches exhibit compromised efficacies [58]. 


\section{Conclusion}

This study introduces a diffusion-assisted approach for the treatment of solid tumors using $\alpha$-particle emitters. To improve the intratumoral $\alpha$-particle emitter distributions for more uniform tumor irradiation, this approach relies on fast-diffusing radioactive species which are released within the tumor interstitium from non-targeted liposomes, following general nanoparticle-based delivery rules for tumor uptake.

\section{Acknowledgements}

Supported in part by the American Cancer Society Research Scholar Grant RSG-12-04401, the National Science Foundation Grant DMR1207022, and the New Jersey Commission on Cancer Research Predoctoral Fellowships (T.L. and M.S.).

Special acknowledgements to Dr Vassiliki Karantza at the Rutgers Cancer Institute of New Jersey for providing us with the transfected cell line, and to Drs George Sgouros and Robert F. Hobbs at Johns Hopkins University for use of the $\alpha$-Camera. We also thank the Radiation Safety Office at Rutgers for assisting with the Cesium-137 irradiator, and Dr Michael Goedken at Rutgers Research Pathology Services for helping with his expertise. 


\section{References}

[1] Bhagat M, Halligan S, Sofou S. Nanoparticles as drug carriers to solid tumors: considerations on tumor penetration and exposure of tumor cells to therapeutic agents. Current Pharm Biotechnology. 2012;13:1306-1316.

[2] Thurber GM, Zajic SC, Wittrup KD. Theoretic Criteria for Antibody Penetration into Solid Tumors and Micrometastases. J Nucl Med. 2007;48:995-999.

[3] Zhu X, Palmer MR, Makrigiorgos GM, Kassis AI. Solid-tumor radionuclide therapy dosimetry: New paradigms in view of tumor microenvironment and angiogenesis. Medical Physics. 2010;37:29742984.

[4] Sgouros G. Alpha-particles for targeted therapy. Advanced Drug Delivery Reviews. 2008;60:1402-1406.

[5] Humm JL. A microdosimetric model of astatine-211 labeled antibodies for radioimmunotherapy. Int J Radiat Oncol Biol Phys. 1987;13:1767-1773.

[6] Humm JL, Chin LM. A model of cell inactivation by alpha-particle internal emitters. Radiat Res. 1993;134:143-150.

[7] Macklis RM, Kinsey BM, Kassis AI, et al. Radioimmunotherapy with alpha-particle-emitting immunoconjugates. Science. 1988;240:1024-1026.

[8] Guerra Liberal FDC, Tavares AAS, Tavares JMRS. Comparative analysis of 11 different radioisotopes for palliative treatment of bone metastases by computational methods. Medical Physics. 2014;41:114101-n/a.

[9] Tavares AAS, Tavares JMRS. Computational Modeling of Cellular Effects Post-Irradiation with Low- and High-Let Particles and Different Absorbed Doses. Dose-Response. 2013;11:191-206.

[10] Fournier C, Zahnreich S, Kraft D, et al. The Fate of a Normal Human Cell Traversed by a Single Charged Particle. Scientific Reports. 2012;2:643.

[11] Allen BJ, Marcu LG, Berak E. Targeted Alpha Therapy for Cancer. Advances in Medical Physics. Vol 6; 2016:177-202. 
[12] Sofou S. Radionuclide carriers for targeting of cancer. Int J Nanomed. 2008;3:181-199.

[13] Maeda H. Tumor-selective delivery of macromolecular drugs via the EPR effect: Background and future prospects Bioconj Chem. 2010;21:797-802.

[14] Helmlinger G, Yuan F, Dellian M, Jain RK. Interstitial pH and pO2 gradients in solid tumors in vivo: high-resolution measurements reveal a lack of correlation. Nature medicine. 1997;3:177-182.

[15] Vaupel P, Kallinowski F, Okunieff P. Blood Flow, Oxygen and Nutrient Supply, and Metabolic Microenvironment of Human Tumors: A Review. Cancer Res. 1989;49:6449-6465.

[16] Karve S, Bajagur Kempegowda G, Sofou S. Heterogeneous domains and membrane permeability in phosphatidylcholine- phosphatidic acid rigid vesicles as a function of $\mathrm{pH}$ and lipid chain mismatch Langmuir. 2008;24:5679-5688.

[17] Bandekar A, Sofou S. Floret-shaped solid domains on giant fluid lipid vesicles induced by $\mathrm{pH}$. Langmuir. 2012;28:4113-4122.

[18] Boggs JM. Lipid intermolecular hydrogen bonding: influence on structural organization and membrane function. Biochim Biophys Acta. 1987;906:353-404.

[19] Bajagur Kempegowda G, Karve S, Bandekar A, Adhikari A, Khaimchayev T, Sofou S. pHdependent formation of lipid heterogeneities controls surface topography and binding reactivity in functionalized bilayers. Langmuir. 2009;25:8144-8151.

[20] Bandekar A, Karve S, M.-Y. C, Mu Q, Rotolo J, Sofou S. Antitumor efficacy following the intracellular and interstitial release of liposomal doxorubicin. Biomaterials. 2012;33:4345-4352.

[21] Karve S, Alaouie A, Zhou Y, Rotolo J, Sofou S. The use of pH-triggered leaky heterogeneities on rigid lipid bilayers to improve intracellular trafficking and therapeutic potential of targeted liposomal immunochemotherapy. Biomaterials. 2009;30:6055-6064.

[22] Stras S, Holeran T, Howe A, Sofou S. Interstitial Release of Cisplatin from Triggerable Liposomes Enhances Efficacy Against Triple Negative Breast Cancer Solid Tumor Analogues. Molecular Pharmaceutics. 2016;13:3224-3233.

[23] Apostolidis C, Molinet R, Rasmussen G, Morgenstern A. Production of Ac-225 from Th-229 for Targeted $\alpha$ Therapy. Analytical Chemistry. 2005;77:6288-6291. 
[24] Zielinska B, Apostolidis C, Bruchertseifer F, Morgenstern A. An Improved Method for the Production of Ac-225/Bi-213 from Th-229 for Targeted Alpha Therapy. Solvent Extraction and Ion Exchange. 2007;25:339-349.

[25] Chang M-Y, Seideman J, Sofou S. Enhanced Loading Efficiency and Retention of 225Ac in Rigid Liposomes for Potential Targeted Therapy of Micrometastases. Bioconjugate Chem. 2008;19:12741282.

[26] Bandekar A, Zhu C, Jindal R, Bruchertseifer F, Morgenstern A, Sofou S. Anti-Prostate-Specific Membrane Antigen Liposomes Loaded with 225Ac for Potential Targeted Antivascular $\alpha$-Particle Therapy of Cancer. Journal of Nuclear Medicine. 2014;55:107-114.

[27] Maguire WF, McDevitt MR, Smith-Jones PM, Scheinberg DA. Efficient 1-Step Radiolabeling of Monoclonal Antibodies to High Specific Activity with 225Ac for $\alpha$-Particle Radioimmunotherapy of Cancer. Journal of Nuclear Medicine. 2014;55:1492-1498.

[28] McDevitt MR, Barendswaard E, Ma D, et al. An alpha-Particle Emitting Antibody ([213Bi]J591) for Radioimmunotherapy of Prostate Cancer. Cancer Res. 2000;60:6095-6100.

[29] Sempkowski M, Zhu C, Menzenski M, et al. Triggered ligand clustering on lipid nanoparticles enables selective targeting and killing of untargetable cancer cells: the case for 'sticky patches'. Langmuir. 2016;32:8329-8338.

[30] Cody S, Dubbin P, Beischer A, et al. Intracellular pH Mapping with SNARF-1 and Confocal Microscopy. II: A Quantitative Technique for Living Tissues and Isolated Cells. Micron. 1993;24:573580.

[31] Back T, Jacobsson L. The alpha-camera: a quantitative digital autoradiography technique using a charge-coupled device for ex vivo high-resolution bioimaging of alpha-particles. J Nucle Med. 2010;51:1616-1623.

[32] Chouin N, Lindegren S, Frost SHL, et al. Ex Vivo Activity Quantification in Micrometastases at the Cellular Scale Using the $\alpha$-Camera Technique. Journal of Nuclear Medicine. 2013;54:1347-1353.

[33] Ballangrud Å, Yang W-H, Palm S, et al. Alpha-particle Emitting Atomic Generator (Actinium225)-Labeled Trastuzumab (Herceptin) Targeting of Breast Cancer Spheroids: Efficacy versus HER2/neu Expression. Clin Cancer Res. 2004;10:4489-4497. 
[34] Graff CP, Wittrup KD. Theoretical analysis of antibody targeting of tumor spheroids: Importance of dosage for penetration and affinity for retention. Cancer Research. 2003;63:1288-1296.

[35] Kamada T, Tsujii H, Blakely EA, et al. Carbon ion radiotherapy in Japan: an assessment of 20 years of clinical experience. The Lancet Oncology.16:e93-e100.

[36] Loeffler JS, Durante M. Charged particle therapy -- optimization, challenges and future directions. Nat Rev Clin Oncol. 2013;10:411-424.

[37] Cooks T, Schmidt M, Bittan H, et al. Local Control of Lung Derived Tumors by Diffusing AlphaEmitting Atoms Released From Intratumoral Wires Loaded With Radium-224. International Journal of Radiation Oncology • Biology • Physics.74:966-973.

[38] Reitkopf-Brodutch S, Confino H, Schmidt M, et al. Ablation of experimental colon cancer by intratumoral 224Radium-loaded wires is mediated by alpha particles released from atoms which spread in the tumor and can be augmented by chemotherapy. International Journal of Radiation Biology. 2015;91:179-186.

[39] Estrella V, Chen T, Lloyd M, et al. Acidity Generated by the Tumor Microenvironment Drives Local Invasion. Cancer Research. 2013;73:1524-1535.

[40] Vaupel P. Tumor microenvironmental physiology and its implications for radiation oncology. Semin Radiat Oncol. 2004;14:198-206.

[41] Brusa P, Immordino ML, Rocco F, Cattel L. Antitumor Activity and Pharmacokinetics of Liposomes Containing Lipophilic Gemcitabine Prodrugs. Anticancer Research. 2007;27:195-199.

[42] Lin YS, Nguyen C, Mendoza J-L, et al. Preclinical Pharmacokinetics, Interspecies Scaling, and Tissue Distribution of a Humanized Monoclonal Antibody against Vascular Endothelial Growth Factor. Journal of Pharmacology and Experimental Therapeutics. 1999;288:371-378.

[43] Miederer M, Scheinberg DA, McDevitt MR. Realizing the potential of the Actinium-225 radionuclide generator in targeted alpha-particle therapy applications. Advanced drug delivery reviews. 2008;60:1371-1382.

[44] Palm S, Baeck T, Elgqvist J, et al. Biodistribution of free ${ }^{225}$ Ac in nude mice. Cancer Biother Radiopharm. 2006;21. 
[45] Sofou S, Thomas JL, Lin H-Y, McDevitt MR, Scheinberg DA, Sgouros G. Engineered liposomes for potential $\alpha$-particle therapy of metastatic cancer. J Nucl Med. 2004;45:253-260.

[46] McDevitt MR, Ma D, Lai LT, et al. Tumor therapy with targeted atomic nanogenerators. Science. 2001;294:1537-1540.

[47] Jaggi J, Kappel BJ, McDevitt MR, et al. Efforts to control the errant products of a targeted in vivo generator. Cancer Res. 2005;65:4888-4895.

[48] Jurcic JG, Levy MY, Park JH, et al. Phase I Trial of Targeted Alpha-Particle Therapy with Actinium-225 $\left({ }^{225} \mathrm{Ac}\right)$-Lintuzumab and Low-Dose Cytarabine (LDAC) in Patients Age 60 or Older with Untreated Acute Myeloid Leukemia (AML). Blood. 2016;128:4050-4050.

[49] Goldenberg DM. Targeted therapy of cancer with radiolabeled antibodies. J Nucl Med. 2002;43:693-713.

[50] Perche F, Torchilin VP. Recent Trends in Multifunctional Liposomal Nanocarriers for Enhanced Tumor Targeting. Journal of Drug Delivery. 2013;2013:705265. doi: 10.1155/2013/705265.

[51] Sofou S, Sgouros G. Antibody-targeted liposomes in cancer therapy and imaging. Expert Opinion on Drug Delivery. 2008;5:189-204.

[52] Prabhakar U, Blakey DC, Maeda H, et al. Challenges and key considerations of the enhanced permeability and retention effect (EPR) for nanomedicine drug delivery in oncology. Cancer Research. 2013;73:2412-2417.

[53] Hansen AE, Petersen AL, Henriksen JR, et al. Positron Emission Tomography Based Elucidation of the Enhanced Permeability and Retention Effect in Dogs with Cancer Using Copper-64 Liposomes. ACS Nano. 2015;9:6985-6995.

[54] Wang AZ. EPR or no EPR? The billion-dollar question. Science Translational Medicine. 2015;7:294ec112-294ec112.

[55] Shi J, Kantoff PW, Wooster R, Farokhzad OC. Cancer nanomedicine: progress, challenges and opportunities. Nat Rev Cancer. 2017;17:20-37.

[56] Basu S, Chen W, Tchou J, et al. Comparison of triple-negative and estrogen receptorpositive/progesterone receptor-positive/HER2-negative breast carcinoma using quantitative fluorine-18 fluorodeoxyglucose/positron emission tomography imaging parameters. Cancer. 2008;112:995-1000. 
[57] Gillies RJ, Robey I, Gatenby RA. Causes and Consequences of Increased Glucose Metabolism of Cancers. Journal of Nuclear Medicine. 2008;49:24S-42S.

[58] Martelotto LG, Ng CKY, Piscuoglio S, Weigelt B, Reis-Filho JS. Breast cancer intra-tumor heterogeneity. Breast Cancer Research : BCR. 2014;16:210.

[59] Bandekar A, Zhu C, Menzenski MZ, Gomez A, Sempkowski M, Sofou S. Masking and triggered unmasking of targeting ligands on liposomal chemotherapy selectively suppress tumor growth in vivo. Mol Pharm. 2013;10:152-160. 

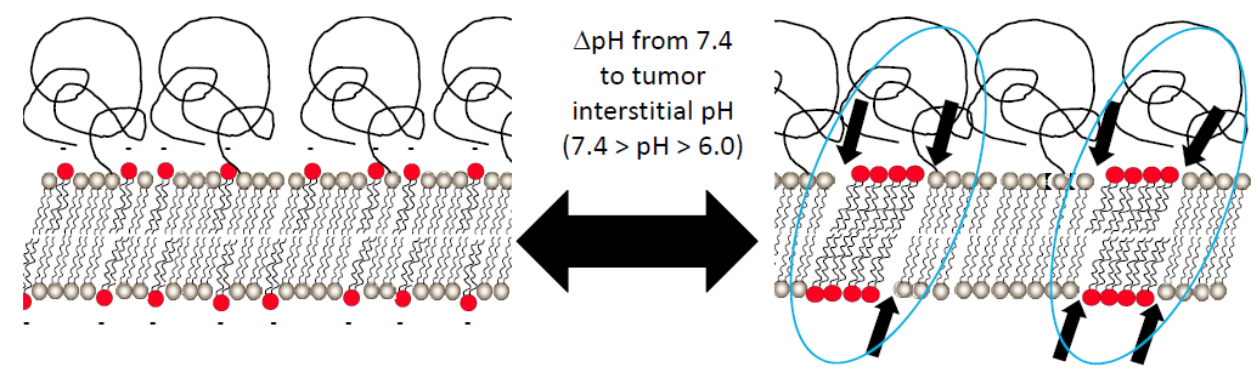

Figure 1. pH-triggered release of contents. (left) $\mathrm{pH}=7.4$ during circulation in blood, (right) at tumor interstitial pH. The upper lipid leaflet represents the outer lipid leaflet of liposomes; the lower lipid leaflet represents the inner lipid leaflet of liposomes. Lipid phase separation and domain formation is activated at the tumor interstitial $\mathrm{pH}(7.4>$ $\mathrm{pH}>6.0$, shown on the right). Black arrows indicate 'leaky' phase boundaries between the different lipid phases. 

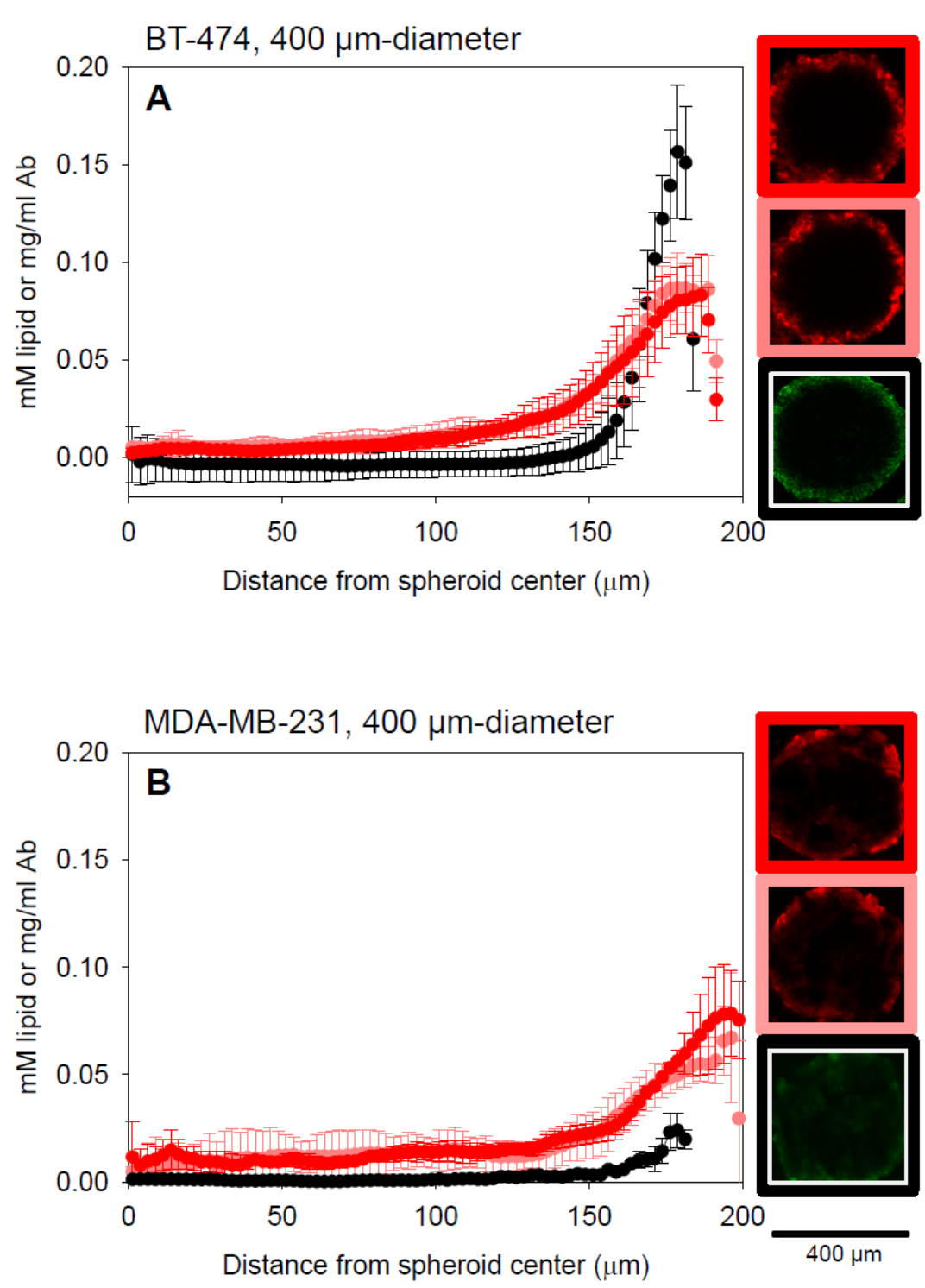

Figure 2. Carrier distributions. Radial distributions of pH-responsive liposomes (red) and non-pH-responsive liposomes (light red) (labeled with fluorescent lipids), and of the HER2-targeting FITC-labeled antibody (black) within $400 \mu \mathrm{m}$-in-diameter spheroids of (A) HER2-positive BT474 cancer cells, and (B) HER2-negative MDA-MB-231 TNBC cells following 6 hour incubation. On the right, characteristic images of the $20 \mu \mathrm{m}$-inthickness equatorial spheroid slices. Errors correspond to standard deviations of $n$ independent spheroids $(\mathrm{n}=5)$. Spheroid diameters varied up to approximately $10 \%$. Immunoreactivity of the FITC-labeled antibody was: $85.1 \pm 7.8 \%$ against HER2-positive BT474 cancer cells. 


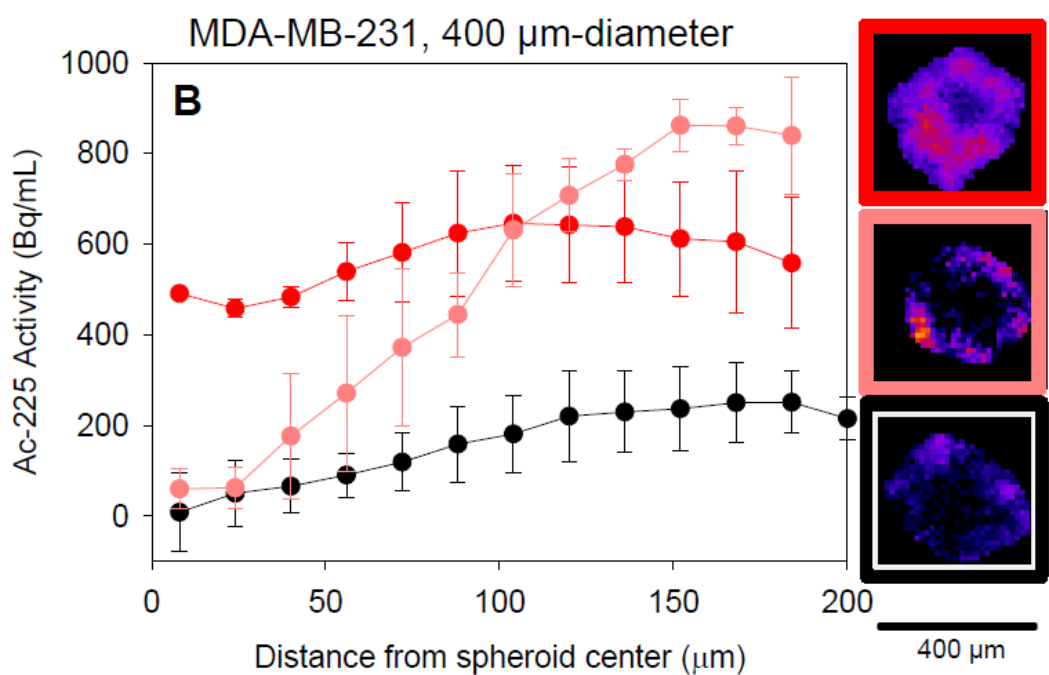

Figure 3. ${ }^{225}$ Ac distributions. Radial distributions and characteristic ( $\alpha$-camera) images (of $8 \mu \mathrm{m}$-in-thickness equatorial $400-\mu \mathrm{m}$-in-diameter spheroid slices) of the ${ }^{225} \mathrm{Ac}$ delivered by $\mathrm{pH}-$ responsive liposomes (red), non-pH-responsive liposomes (light red), and of the HER2-targeting radiolabeled antibody (black) within spheroids of (A) HER2positive BT474 cancer cells, and (B) HER2-negative MDA-MB-231 TNBC cells. Activities were corrected for radioactive decay. Image pixel intensities were corrected for radioactive decay. Spheroids were incubated with $462.5-925 \mathrm{kBq} / \mathrm{mL}(12.5-25$ $\mu \mathrm{Ci} / \mathrm{mL}$ ) for 6 hours. Errors correspond to standard deviations of $n$ independent spheroids $(n=5)$. Spheroid diameters varied up to approximately $10 \%$. 


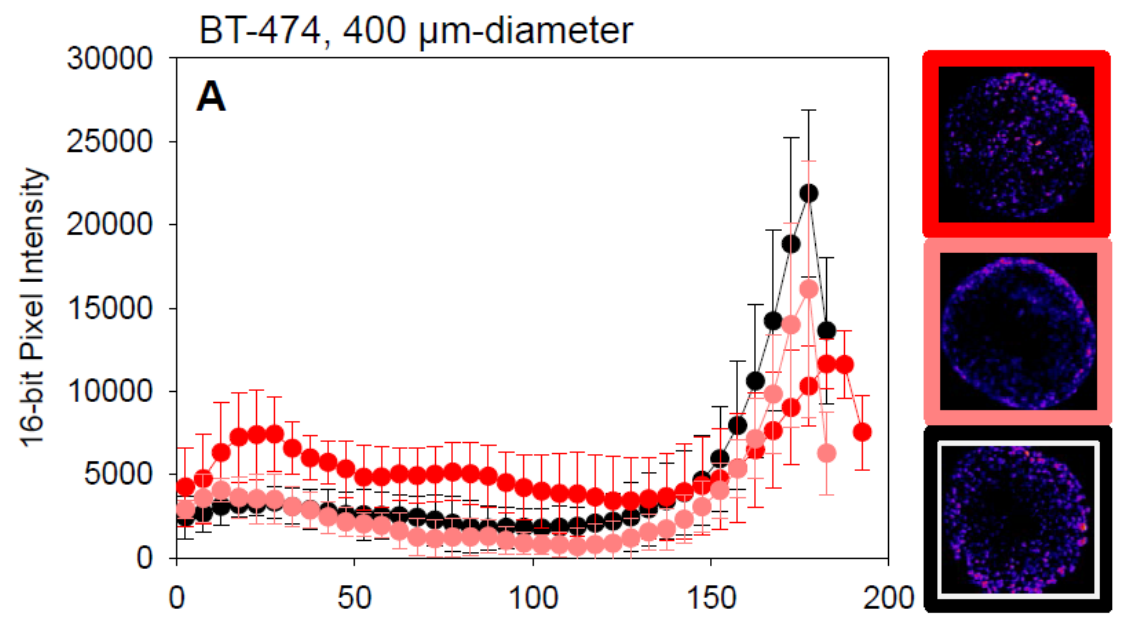

Distance from spheroid center $(\mu \mathrm{m})$

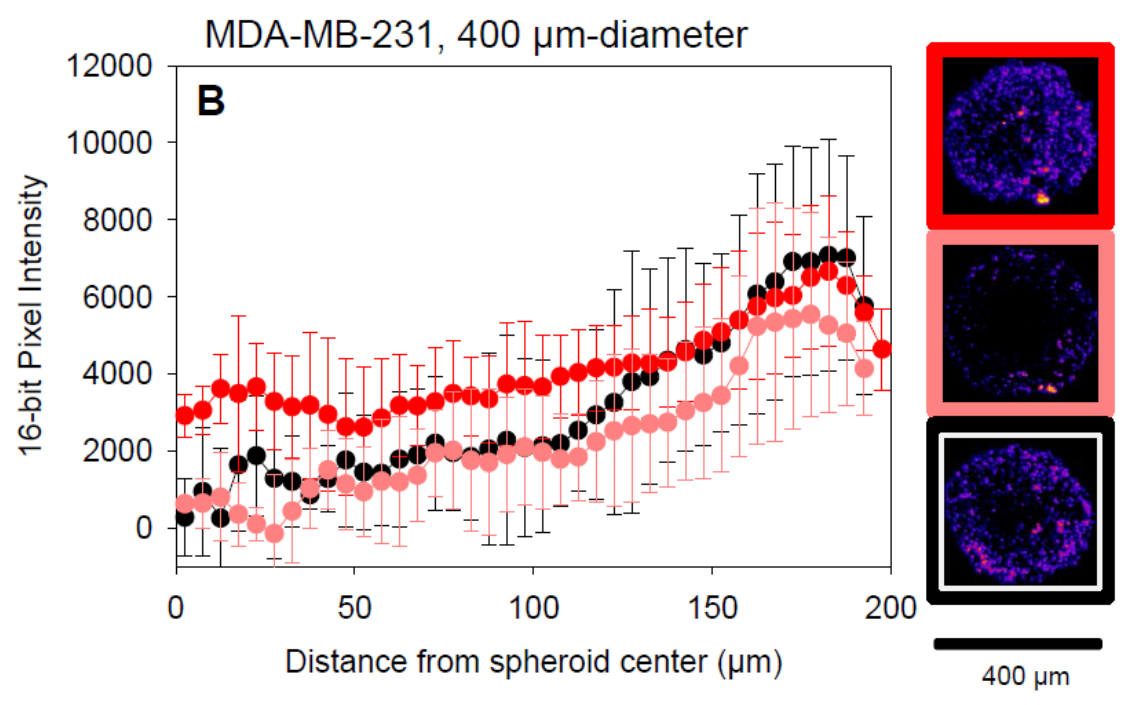

Figure 4. Radial distributions of $\gamma-\mathbf{H 2 A X}$ foci in spheroid slices treated with immunofluorescent staining for $\gamma-\mathrm{H} 2 \mathrm{AX}$ caused by the delivered radioactivity by $\mathrm{pH}-$ responsive liposomes (red), non-pH-responsive liposomes (light red), and the HER2targeting radiolabeled antibody (black) in $400 \mu \mathrm{m}$-in-diameter spheroids of (A) HER2positive BT474 cancer cells, and (B) HER2-negative MDA-MB-231 TNBC cells. Errors correspond to standard deviations of $n$ independent spheroids ( $n=6$ for BT-474 and 3 for MDA-MB-231). Spheroids were incubated with $92.5 \mathrm{kBq} / \mathrm{mL}(2.5 \mu \mathrm{Ci} / \mathrm{mL})$ radioactivity for 6 hours. On the right, characteristic images of $20 \mu \mathrm{m}$-in-thickness equatorial spheroid slices. Spheroid diameters varied up to approximately $10 \%$. 

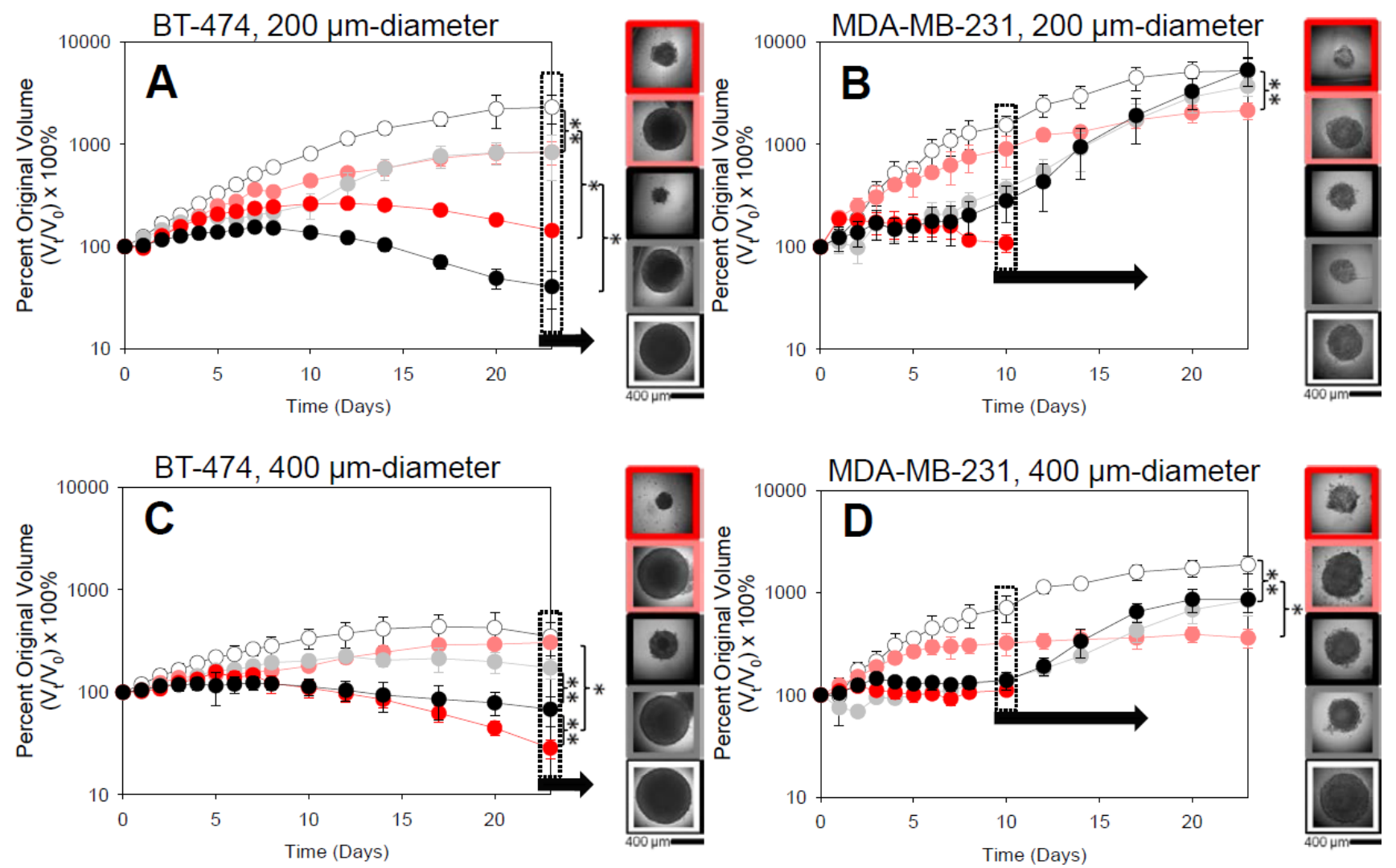

Figure 5. Growth control of 'small' 200- $\mu$ m-in-diameter spheroids of (A) HER2-overexpressing BT474 cells and (B) TNBC MDA-MB-231 cells, and of 'large' 400- $\mu$ m-in-diameter spheroids of (C) BT474 cells and (D) MDA-MB-231 cells treated for 6 hours with18.50 kBq/mL (500 nCi/mL) delivered by pHreleasing liposomes (red), non-pH-releasing liposomes (light red), a HER2-targeting radiolabeled antibody (black) and a radiolabeled isotype control antibody (gray). 'No treatment' is shown in white. Growth control in the presence of non-radiolabeled carriers is shown in Figures S15-21. Errors correspond to standard deviations of repeated measurements ( $n=3$ independent liposome or antibody preparations, 5-8 spheroids per type of carrier per preparation). On the right, characteristic bright field images of spheroids. Spheroid diameters at time of treatment varied up to approximately $10 \%$. ${ }^{*} p$-values $<0.01, * * p$-values $<0.05$. 
Figure 6. (A) animal survival and (B) growth of orthotopic HER2-negative MDA-MB231 tumors following one i.v. administration when tumors reached $100 \mathrm{~mm}^{3}$ of $9.25 \mathrm{kBq}$ $\left(250 \mathrm{nCi}\right.$ ) of ${ }^{225} \mathrm{Ac}-$ loaded $\mathrm{pH}-$ responsive liposomes (red, $\left.n=8\right),{ }^{225} \mathrm{Ac}-$ loaded non-pHresponsive liposomes (light red, $n=8$ ), and of the HER2-targeting radiolabeled antibody Trastuzumab (black, $n=8$ ). Animal response to non-radiolabeled liposomes was indistinguishable from no-treatment (saline; white symbols, $n=6$ ) [59]. Error bars correspond to standard errors of the mean. ${ }^{* *} p<0.05$ using the Kaplan-Meier method (log-rank test); ${ }^{*} p<0.01$ by Student's t-test for tumor volumes. 
Table 1

Characterization of $\mathrm{pH}-$ responsive and non-pH-responsive liposomes. Errors correspond to standard deviations of $n$ independent liposome preparations.

\begin{tabular}{|c|c|c|c|c|c|}
\hline $\begin{array}{c}\text { Nanocarrier } \\
\text { type }\end{array}$ & $\begin{array}{c}\text { Size, nm } \\
\text { (PDI) } \\
(n)\end{array}$ & $\begin{array}{c}\text { Zeta } \\
\text { potential, } \\
\mathrm{mV} \\
(n)\end{array}$ & $\begin{array}{l}\text { \% Loading } \\
\text { efficiency } \\
\quad(n)\end{array}$ & $\begin{array}{c}\text { Radioactivity } \\
\text { retention in } \\
\text { serum after } 24 \\
\text { hours at } \\
\mathrm{pH}=7.4 \\
\text { (n) }\end{array}$ & $\begin{array}{c}\text { Radioactivity } \\
\text { retention in } \\
\text { serum after } 6 \\
\text { hours at } \\
\mathrm{pH}=6.0 \\
(n)\end{array}$ \\
\hline $\begin{array}{c}\text { pH-responsive } \\
\text { liposomes }\end{array}$ & $\begin{array}{c}114 \pm 8 \\
(0.09 \pm 0.03) \\
(n=6)\end{array}$ & $\begin{array}{c}-6.8 \pm 2.3 \\
(n=6)\end{array}$ & $\begin{array}{c}43.5 \pm 12.8 \\
(n=6)\end{array}$ & $\begin{array}{c}77.6 \pm 6.2 \\
(n=6)\end{array}$ & $48.6 \pm 5.8$ \\
\hline $\begin{array}{l}\text { non-pH- } \\
\text { responsive } \\
\text { liposomes }\end{array}$ & $\begin{array}{c}108 \pm 15 \\
(0.12 \pm 0.04) \\
(n=6)\end{array}$ & $\begin{array}{c}-0.8 \pm 2.8 \\
(n=6)\end{array}$ & $\begin{array}{c}61.0 \pm 10.1 \\
(n=6)\end{array}$ & $\begin{array}{c}81.8 \pm 4.5 \\
(n=6)\end{array}$ & $\begin{array}{c}78.8 \pm 4.6 \\
(n=6)\end{array}$ \\
\hline
\end{tabular}




\section{Table 2}

Characterization of radiolabeled antibodies. Errors correspond to standard deviations of $n$ independent preparations.

\begin{tabular}{|c|c|c|c|c|}
\hline $\begin{array}{c}\text { Nanocarrier } \\
\text { type }\end{array}$ & $\begin{array}{l}\text { \% Loading } \\
\text { efficiency } \\
(n)\end{array}$ & $\begin{array}{c}\text { Radiochemical } \\
\text { purity } \% \\
(n)\end{array}$ & $\begin{array}{c}\% \\
\text { Immunoreactivity } \\
(n)\end{array}$ & $\begin{array}{c}\text { Radioactivity } \\
\text { retention in } \\
\text { serum after } \\
24 \text { hours at } \\
\mathrm{pH}=7.4 \\
(n)\end{array}$ \\
\hline $\begin{array}{c}\text { HER2- } \\
\text { targeting Ab }\end{array}$ & $\begin{array}{c}70.1 \pm 17.6 \\
(n=6)\end{array}$ & $\begin{array}{c}95.6 \pm 1.9 \\
(n=6)\end{array}$ & $\begin{array}{c}86.5 \pm 6.2 \\
(n=6)\end{array}$ & $\begin{array}{c}88.2 \pm 4.4 \\
(n=5)\end{array}$ \\
\hline $\begin{array}{l}\text { Isotype- } \\
\text { control Ab }\end{array}$ & $\begin{array}{c}54.1 \pm 21.0 \\
(n=4)\end{array}$ & $\begin{array}{c}97.2 \pm 1.1 \\
(n=4)\end{array}$ & $\begin{array}{c}1.0 \pm 0.8 \\
(n=4)\end{array}$ & $\begin{array}{c}82.8 \pm 5.9 \\
(n=4)\end{array}$ \\
\hline
\end{tabular}




\section{Table 3}

Measured expression of HER2 copies per cell using cells harvested from monolayers.

Errors correspond to standard deviations of $n$ independent measurements.

\begin{tabular}{|l|l|}
\hline Cell Line & HER2 copies per cell $(n)$ \\
\hline BT474 & $1.3 \pm 0.1 \times 10^{6}(n=5)$ \\
\hline MCF-7/HER2 & $1.6 \pm 0.4 \times 10^{5}(n=2)$ \\
\hline MDA-MB-231 & $8.3 \pm 0.8 \times 10^{4}(n=3)$ \\
\hline
\end{tabular}

